\title{
Gradient Based Image Edge Detection
}

\author{
Jamil A. M. Saif, Mahgoub H. Hammad, and Ibrahim A. A. Alqubati
}

\begin{abstract}
Due to the importance of image edge detection in image analysis, object recognition and many applications, many edge detection algorithms are used to detect edges of objects in the image. Edges typically occur on the boundary between two different regions in the image. There are a number of algorithms for this, but these may be classified as derivative based where the algorithm takes first or second derivative on each pixel, or gradient based where a gradient of consecutive pixels is taken in $x$ and $y$ direction.

In our paper we address the problem of gradient based image edge detection, several algorithms are tested, as a result of these algorithms binary images are produced, which represent objects and their background which then helps interpreting the content of the considered images, several medical(for different and the same organ) as well as natural images are used to evaluate the performance of the algorithms and their suitability for both kinds of images.
\end{abstract}

Index Terms - Canny edge detection, image analysis, image edge detection.

\section{INTRODUCTION}

The problem of Image edge detection have been known and studied intensively for the last three decades, and surely plays an important role in image analysis and computer vision systems. But, it still considered to be one of the most difficult and challenging tasks in image processing and object recognition, that determines the quality of final results of the image analysis. In image processing [1]-[5] Image edge detection is a basic problem in spectrum of applications [2], [6]-[8]. It's the process of converting a grey scale image into binary image, which is based on discontinuity search for abrupt changes in the intensity value, such methods are called edge or boundary based methods [3], [9], [10]. They detect discontinuities and produce a binary images contained edges and their background as the output of them. Edges are local changes in the image intensity, they typically occur on the boundary between two regions, important features can be extracted from these edges, then The features are used by higher level computer vision algorithms [1], [2], [11], [12]. Edge detection is used for object detection, recognition and many other applications. It is an active area of research as it facilitates higher level of image analysis. A number of edge detectors based on a single derivative have been developed by various researchers [3], [9], [13], [14]. In this paper we will use Sobel, Prewitt and Canny filters for our experiments to investigate their performance.

Manuscript received September 16, 2014; revised December 18, 2014.

Jamil A. M. Saif and Mahgoub H. Hammad are with the Information Systems Dept, Community College of Bisha, King Khalid University, KSA, Saudi Arabia (e-mail: jamil_alabssi@yahoo.com,mhammad@kku.edu.sa).

Ibrahim A. A. Alqubati is with the Information Systems Dept, Community College of Najran, Najran University, KSA, Saudi Arabia (e-mail: alqubati_ibr@yahoo.com).
The contents of this paper is organized as follows: in Section II the proposed algorithms and their descriptions are presented, in Section III the experimental results are presented and discussed, conclusion and recommendation for future work is given in Section IV.

\section{THE PROPOSED ALgORITHMS}

Many edge detection algorithms are used [7], [14] to detect edges of objects in the image. Edges typically occur on the boundary between two different regions in the image. There are a number of algorithms for this, but these may be classified as derivative based where the algorithm takes first or second derivative on each pixel, or gradient based where a gradient of consecutive pixels is taken in $x$ and $y$ direction. An operation called kernel operation is usually carried out. A kernel is a small matrix centered on a chosen pixel of the image matrix, multiplied the coefficients of the filter with the corresponding pixels of image matrix for the specified pixel located on the centre of the matrix, if the calculated value is above a specified threshold, then the middle pixel is classified as an edge, and such calculation is repeated for each pixel of the image [13], [15], sliding over the image matrix from left to right and from up down. Sobel, Prewitt and Canny are examples of gradient based methods of edge detection.

\section{A. Sobel and Prewitt Algorithms}

Sobel and Prewitt algorithms are widely used for image edge detection and segmentation [8], [16]-[19]. The kernels of such algorithms for $\mathrm{x}$ and $\mathrm{y}$ directions are presented in Fig. 1.
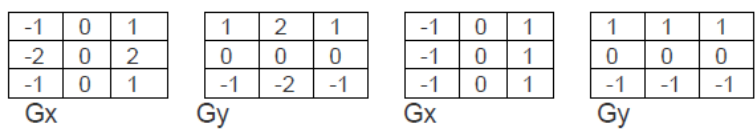

a)

b)

Fig. 1. kernels of a) Sobel b) Prewitt.

The edge detection operation is essentially an operation to detect significant local changes in the intensity level in an image. The change in intensity level is measured by the gradient of the image. Since an image $f(x, y)$ is a two-dimensional function, its gradient is a vector. The magnitude and the direction of the gradient may be computed as given by the formulae 1 and 2 respectively:

$$
\begin{aligned}
& |G|=\sqrt{G_{x}^{2}+G_{y}^{2}} \\
& \infty(x, y)=\tan ^{-1}\left(\frac{G_{y}}{G_{x}}\right)
\end{aligned}
$$




\section{B. Canny Edge Detector}

The Canny edge detector is regarded as one of the best edge detectors currently in use, Canny's edge detector ensures good noise immunity and at the same time detects true edge points with minimum error.

Canny has optimized the edge detection with regard to the following criteria:

1) Maximizing the signal-to-noise ratio of the gradient, so that the probability of detecting real edge points should be maximized while the probability of falsely detecting non-edge points should be minimized.

2) An edge localization factor, which ensures that the detected edge is localized as accurately as possible.

3) Minimizing multiple responses to a single edge, so thould not result in more than one detected edge.

The steps of Canny algorithm [12], [20]-[24] are as follows:

1) Smoothing: Blurring of the image to remove noise by convolving the image with the Gaussian filter.

2) Finding gradients: The edges should be marked where the gradients of the image has large magnitudes, finding the gradient of the image by feeding the smoothed image through a convolution operation with the derivative of the Gaussian in both the vertical and horizontal directions

3) Non-maximum suppression: Only local maxims should be marked as edges. Finds the local maxima in the direction of the gradient, and suppresses all others, minimizing false edges.

4) Double thresholding: Potential edges are determined by thresholding, Instead of using a single static threshold value for the entire image, the Canny algorithm introduced hysteresis thresholding, which has some adaptivity to the local content of the image. There are two threshold levels, $t h$, high and $t_{1}$, low where $t h>t_{1}$. Pixel values above the th value are immediately classified as edges.

5) Edge tracking by hysteresis: Final edges are determined by suppressing all edges that are not connected to a very strong edge.

\section{EXPERIMENTAL RESULTS}

The paper presents three of gradient based image edge detection algorithms, as examples Sobel, Prewitt and Canny detectors are considered. They are tested with a variety of representing medical as well as natural images and their corresponding edge images, many experiments were investigated with several images, below we show six images and their edges in Fig. 2, Fig. 3 and Fig. 4, where Fig. 2 shows the results of non medical images, and the results of different organs of medical (MRI and Endoscopic) images are shown in Fig. 3 and for similar medical (only Endoscopic) images are shown in Fig. 4. The strategy of using different images give us a wider explanation and allow to specify the suitability of the test algorithms regarding the kind as well as the quality of images, however it is very difficult to distinguish endoscopic image of healthy organ from that one with a disease.
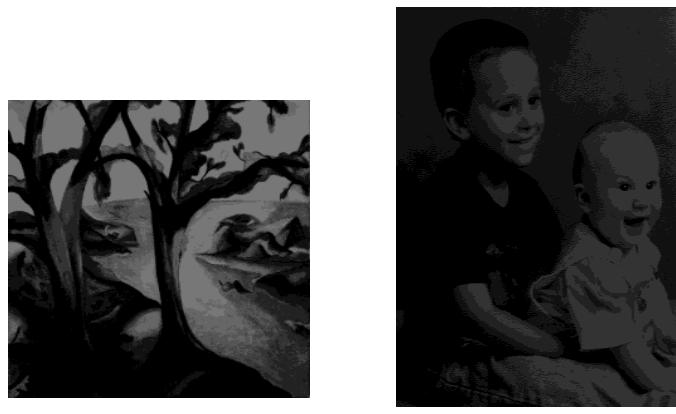

a)
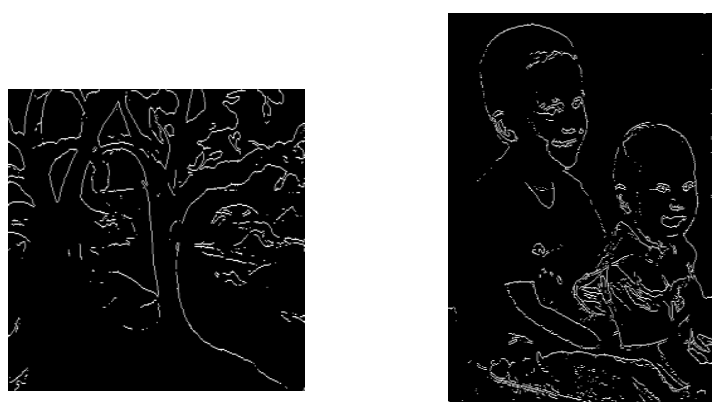

b)
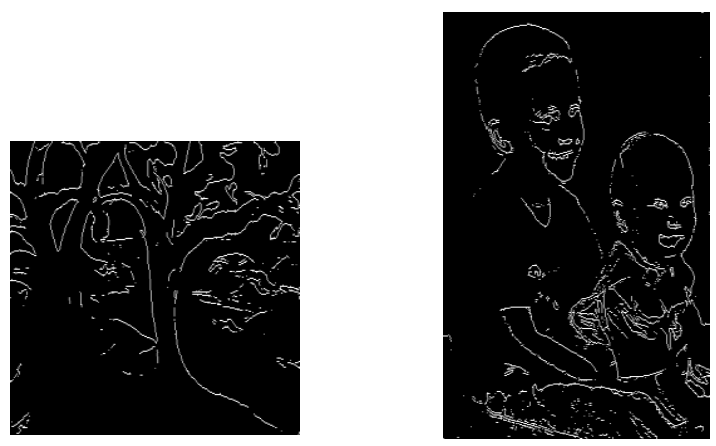

c)
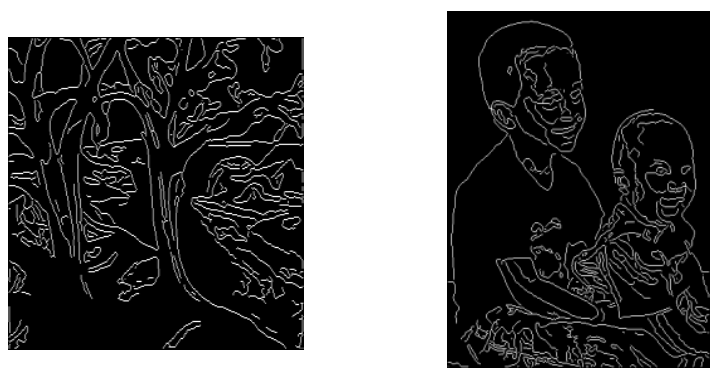

d)

Fig. 2. a) Original images. b) their Sobel edge images. c) their Prewitt edge images. d) their Canny edge images.
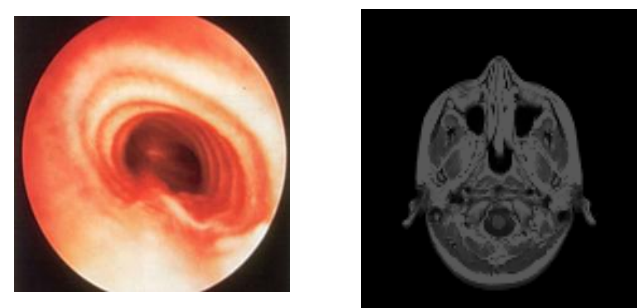

a) 

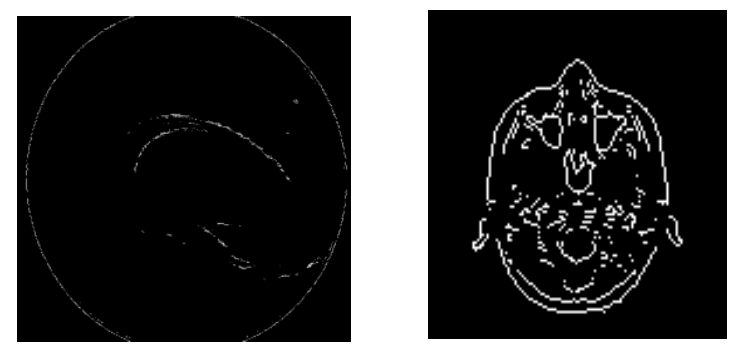

b)
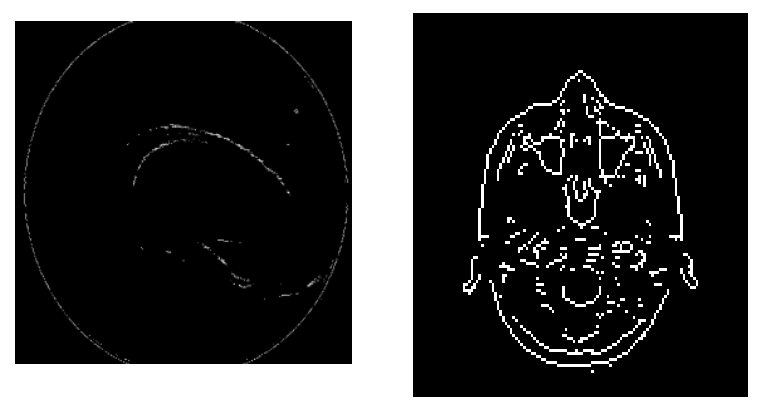

c)
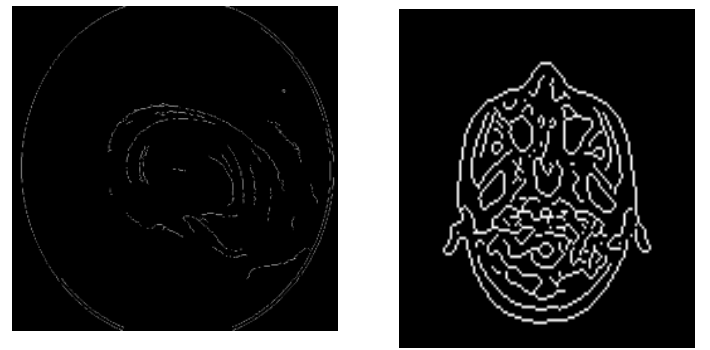

d)

Fig. 3. a) Original images. c) their Prewitt edge images.
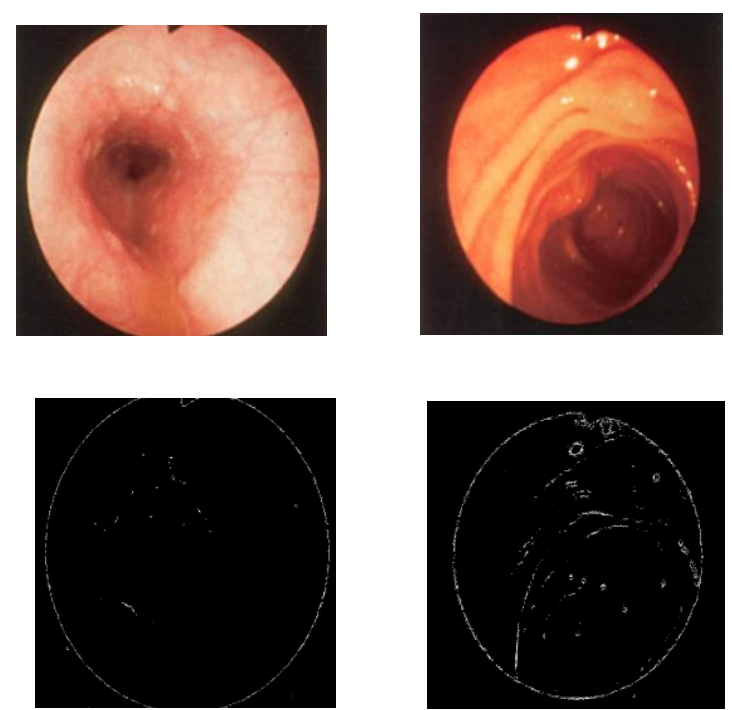

b)
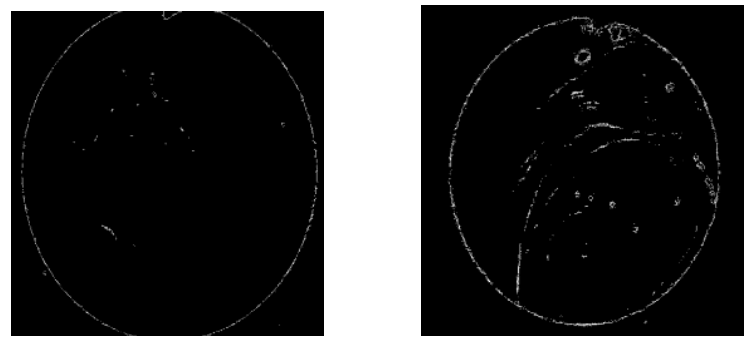
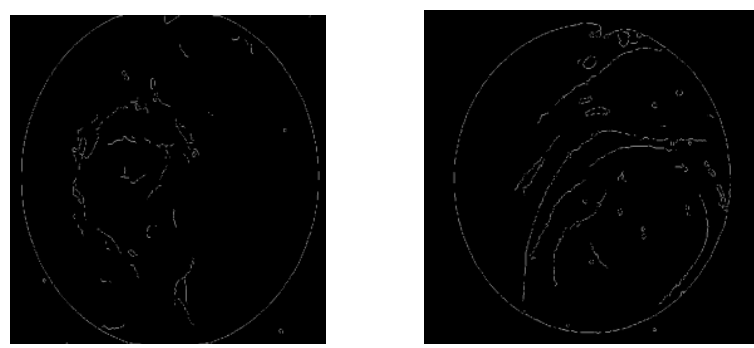

d)

Fig. 4. a) Original images. b) their Sobel edge images. c) their Prewitt edge images. d) their Canny edge images.

\section{CONCLUSION}

Image edge detection is a challenging task due to the noises and the nature of images, so a preprocessing step is required for image edge detection, that may reduce the noises and enhance the result, because any deficiency during the image acquisition can cause many problems that affects the result of edge images, and consequently affects image analysis.

The results presented above show that the efficiency of the algorithms are dependable on the type of images and their application and their contents.

In this paper the effectiveness of the proposed algorithms are evaluated for medical(MRI and Endoscopic) and non medical images, for non medical images as shown in Fig. 2, the Sobel and Prewitt algorithms give better results than Canny algorithm, on the other hand Canny algorithm is more suitable for medical images than Sobel and Prewitt as it shown in Fig. 3 and 4, where the objects are less distinguished from their background, but for color endoscopic image and for MRI grey scale image as it is shown in Fig. 3, Canny produces too many no needed edges, but for endoscopic images as it shown in Fig. 4, when the edges are not strong or the objects within the image is not very clear, Canny operator seemed to be more optimal than Sobel and Prewitt.

Finally it is recommended for the future work to test the tuning of Canny parameters, searching the optimal ones (threshold, sigma, etc) that give more effective results and to mange with a variety of images, this can be done using heuristic search, however it is strongly recommended to apply artificial methods, that facilitate detecting edges with the specific optimal Canny parameters. Also it can be tested further algorithms of edge detection and making comparison of their results with currently tested algorithms.

\section{REFERENCES}

[1] T. Acharya and A. K. Ray, Image Processing Principles and Applications, John Wiley \& Sons, Inc. 2005.

[2] B. Chanda and D. D. Majumder, Digital Image Processing and Analysis, Prentice Hall, 2003.

[3] R. C. Gonzalez and R. E. Woods, Digital Image Processing, Second Edition, Prentice Hall, 2002

[4] Summary of Image Edge Detection. [Online]. Available: http://en.cnki.com.cn/Article_en/CJFDTOTAL-GXJS200503027.htm

[5] Flectures. [Online]. Available: http://www.google.com.sa/url?sa=t\&rct=j\&q=\&esrc=s\&source=web $\& \mathrm{~cd}=13 \&$ ved $=0 \mathrm{CGgQFj}$ AM $\& u r l=$ http $\% 3 \mathrm{~A} \% 2 \mathrm{~F} \% 2 \mathrm{Fwww}$. cfar.umd.e du\%2F fer\%2Fcmsc426\%2Flectures\%2Fedge1.ppt\&ei=0YKVVPTZ Es3maIq7gNAK\&usg=AFQjCNGNJ6jrtJejCgNLYQ4LOhOBju3rIw \&sig2=SLGb6dhAOWi3Djw_9T29jw 
[6] G. J. Paul and P. H. Clare, Introductory Remote Sensing Digital Image Processing and Applications, 2000

[7] M. Sonka, V. Hlavac, and R. Boyle, Image Processing, Analysis and Machine Vision, Thomson, 2008

[8] An Adaptive Edge-detection Method Based on Canny Algorithm. [Online].

Available: http://en.cnki.com.cn/Article_en/CJFDTOTAL-ZGTB200408010.htm

[9] J. K. Anil, Fundamentals of Digital Image Processing, Prentice Hall, April, 2004

[10] P. Thakare, "A study of image segmentation and edge detection techniques," International Journal on Computer Science and Engineering(IJCSE), Feb. 2011

[11] S. E. Umbaugh, Computer Vision and Image Processing: A Practical Approach Using CVIP tools, Prentice Hall, 1998

[12] Fourier. [Online]. http://fourier.eng.hmc.edu/e161/lectures/canny/node1.html

[13] H. B. Kekre, T. K. Sarode, and R. Bhakti, "Color image segmentation using Kekre's algorithm for vector quantization," International Journal of Computer Science, vol. 2, 2008

[14] J. A. Madhuri, Digital Image Processing, Prentice Hall, 2006

[15] L. Spirkovsk, A Summary of Image Segmentation Techniques, Ames Research Center, Moffett Field, California, 1993

[16] W. Malina, S. Ablameyko, and W. Pawlak, Fundamental Methods of Digital Image Processing, 2002

[17] J. Saif and A. Moharram, "Edge and regin based image segmentation," Journal of Computer and Information Technology of Hodeidah University, Hodedah, Yemen, 2011

[18] J. Saif, A A. Kubati, A. Hazaa, and M. Almourish, "Image segmentation using edge detection and thresholding," in Proc. the 2012 International Arab Conference on Information Technology, Jordan, Dec. 2012

[19] E. A. Savakis, "Adaptive Document Image Thresholding Using Foreground and Background clustering," in Proc. the International Conference on Image Processing ICIP, vol. 98, 2003.

[20] Canny's Edge Detector: Implementation. [Online]. Available: http://suraj.lums.edu.pk/ cs436a02/CannyImplementation.htm

[21] Canny Edge Detection in C. [Online]. Available:.http://www.codeproject.com/KB/cs/Canny_Edge_Detectio n.aspx

[22] Daftar Pustaka. [Online]. Available: http://www.cvmt.dk/education/teaching/f09/VGIS8/AIP/canny_09gr8 20.pdf

[23] Canny Edge Detection. [Online]. Available: http://docs.opencv.org/trunk/doc/py_tutorials/py_imgproc/py_canny/p y_canny.html

[24] Canny Edge Detection Parameters. [Online]. Available: http://wintopo.com/help/html/canny-opt.htm

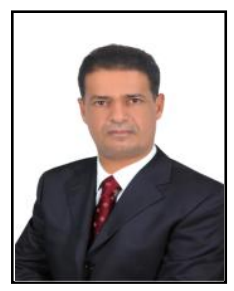

Jamil A. M. Saif received his MSc degree in telecommunication engineering from the Department of telecommunication, Faculty of Automatic Control Electronics and Computer Science, Silesian University of Technology, Gliwice, Poland, in 1993, He received his $\mathrm{PhD}$ degree in computer science from the Department of Computer Architecture, Faculty of Electronics, telecommunications and Informatics, Gdansk University of Technology, Gdansk, Poland, in 2004. In May 2012 he got promoted to associate professor at the Faculty of Computer Science and Engineering, Hodeidah University, Hodeidah, Yemen From 2013 till now he is working in the Department of Information Systems, Community College of Bisha , King Khalid University, Kingdom of Saudi Arabia.

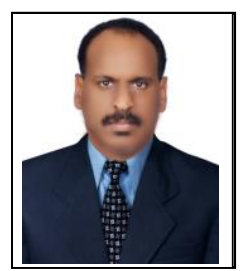

Mahgoub H. Hammad received his BSc and MSc degrees in computer science from the Deparment of Computer Science, Faculty of Mathematical Science, University of Khartoum, Sudan, in 1995 and 2006 respectively. Currently he is the head Department of Information Systems, Community College of Bisha, King Khalid University, Kingdom of Saudi Arabia. At the same time form 2010 to 2014 he is a Phd researcher in computer science at the Department of Computer Science, Faculty of Mathematical Science, University of Khartoum, Sudan. Currently he is preparing to discuss his PhD Thesis.

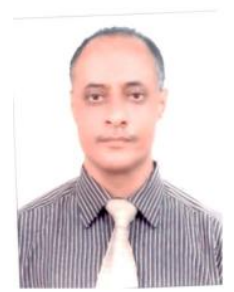

Ibrahim A. A. Alqubati received his MSc degree in computer science from the Department of Software Engineering, Faculty of Informatics and Management, Wroclaw University of Technology, Wroclaw, Poland, in 1990 and He received his PhD degree in artificial intelligence (artificial neural networks) from Wroclaw University of Technology, Wroclaw, Poland, in 1999. From 2000 till 2012 he was the dean of Computer Science and Engineering Faculty of Hodeidah University, Hodeidah, Yemen. In 2005 he got promoted to associate professor, and in 2011 he was promoted to a full professor at the Faculty of Computer Science and Engineering, Hodeidah University, Hodeidah, Yemen. Currently he is a professor at Community College of Najran University, Najran, Kingdom of Saudi Arabia. In addition he is the head of Department of Information Systems. 